\title{
Retardation mechanism of anionic asphalt emulsion on the hydration
} of Portland cement

\author{
Wei Li ${ }^{1,2,3,4^{*}}$, Jinxiang Hong ${ }^{3}$, Xiaobin $\mathrm{Zhu}^{3}$, Dingyi Yang ${ }^{2}$, Yun Bai ${ }^{4}$, Jiaping Liu ${ }^{1,3,4^{*}}$, Changwen Miao ${ }^{1,3}$ \\ 1. School of Materials Science and Engineering, Southeast University, Nanjing, China. \\ 2. Green Building Material Institute, College of Civil Science and Engineering, Yangzhou University, \\ Yangzhou, China. \\ 3. State Key Laboratory of High Performance Civil Engineering Materials, Nanjing, China. \\ 4. Advanced \& Innovative Materials (AIM) Group,University College London, London, UK.
}

\begin{abstract}
Cement-asphalt (CA) mortar, a grouting material consisting of Portland cement (PC), asphalt
\end{abstract} emulsion, sand, water and other related admixtures, has been widely used as a cushion layer material in the construction of High-Speed Railways (HSR) during the past decade in China due to its excellent damping property. The fresh and hardened properties of CA mortar are closely related to the PC hydration in the presence of asphalt emulsion. However, the retardation effect introduced by the anionic asphalt emulsion on the hydration process of PC has not been fully understood. In this paper, the effect of an anionic asphalt emulsion on the early hydration process of CA paste was first investigated by setting time test, isothermal conduction calorimeter and electrical resistivity. The MIP and SEM were then employed to characterize the microstructure evolution of the CA paste. Based on the data and observation obtained from the experimental study, three possible retardation mechanisms introduced by the anionic asphalt emulsion on the PC hydration were then assessed in this paper, including (i) selective adsorption by anionic emulsifier via electrostatic attraction, (ii) coating formed by the demulsification of anionic asphalt emulsion, and (iii) reduced reactivity of water molecules in anionic asphalt emulsion.

Key Words: Anionic asphalt emulsion; Cement hydration; Retardation; Water Reactivity.

\footnotetext{
Corresponding author at : School of Materials Science and Engineering, Southeast University. Email:230129319@seu.edu.cn
} 


\section{Introduction}

Cement-based materials have been widely used in civil engineering because of their superb compressive strength and locally available supply of raw materials. However, the brittleness incurred from the high modulus of elasticity of cement-based materials have hindered their applications in certain areas where some level of ductility, in particular, damping property, is required [1-5]. Asphalt is a flexible material with excellent deformation capacity. However, its low compressive strength forms a barrier for some applications [6-7].

As a kind of inorganic-organic composite, cement-asphalt (hereafter CA) mortar, which consists of Portland cement, asphalt emulsion, water and other related admixtures, is able to combine the high compressive strength of cement and the superb flexibility of asphalt [8-11]. As a result, in recent decades, CA mortar has been extensively used as a grouting material in the cushion layer of the slab track system of High-Speed Railways (HSR) in China, primarily due to its excellent damping property $[12-15]$

In the formulation of CA mortar, asphalt emulsion, instead of asphalt, has to be adopted to modify the properties of PC paste, as this can ensure a good compatibility to be achieved between the cement particles and the asphalt molecules. In general, two kinds of asphalt emulsion, including cationic and anionic asphalt emulsions, can be employed to formulate the CA mortars, and the properties of CA mortar can be strongly affected by the type of asphalt emulsion used. This is because the hydration of silicate phases, such as $\mathrm{C}_{3} \mathrm{~S}$ and $\mathrm{C}_{2} \mathrm{~S}$, could be hindered in the presence of cationic asphalt emulsion due to the adsorption of cationic asphalt emulsion onto the negatively charged surface of the silicate phases via electrostatic attraction, leading to a reduction in the strength of CA paste. On the contrary, the anionic asphalt emulsion is primarily adsorbed onto the positively charged surface of aluminate phases, such as $\mathrm{C}_{3} \mathrm{~A}$ and some small parts of silicate phases [16]. Therefore, the anionic asphalt emulsion could only exert some slight hindrances to the hydration of $\mathrm{C}_{3} \mathrm{~S}$ and $\mathrm{C}_{2} \mathrm{~S}$. As it is the hydration of $\mathrm{C}_{3} \mathrm{~S}$ and $\mathrm{C}_{2} \mathrm{~S}$ that mainly contributes to the strength development of PC paste, it could be deduced that anionic asphalt emulsion is more suitable than cationic asphalt emulsion for formulating CA mortars when a higher strength is required (such as the Type II CA Mortar as specified in the current Chinese Standard) [17-19]. However, the effect of anionic asphalt emulsion on the hydration of 
1 PC has not been fully understood. Although some researchers have reported that the anionic asphalt

2 emulsion can retard the hydration of PC [20-22], the retardation mechanism has not been fully explored.

3 Nonetheless, a good understanding of the retardation mechanism introduced by the anionic asphalt emulsion to the hydration of PC is considered essential to the wider industrial application of the CA mortars because it could affect the rheological property and the microstructural evolution of the CA mortar. Therefore, in this paper, an anionic asphalt emulsion was used to formulate a type II CA mortar where a relatively higher strength is required under the current Chinese Standard for High-Speed Railway [19, 23-24]. The effect of this anionic asphalt emulsion on the early hydration process of the PC paste was firstly investigated by setting time test, isothermal conduction calorimetry and electrical resistivity. Additionally, the MIP and SEM were also employed to characterize the microstructure evolution of the CA paste. Based on the data and the observation obtained from aforementioned tests, three possible retardation mechanisms introduced by the anionic asphalt emulsion on the PC hydration were then assessed and discussed.

\section{Raw materials, sample preparation and test methods}

\subsection{Raw materials}

Portland cement (PC), type P.I 42.5, complying with the Chinese standard GB8076-2008, produced by Qufu China United Cement Co., LTD was used in this study. Its chemical composition is presented in Table 1.

Table 1 Composition of Portland cement(wt.\%)

\begin{tabular}{ccccccccc}
\hline \multicolumn{7}{c}{ Chemical composition } \\
\hline $\mathrm{SiO}_{2}$ & $\mathrm{Fe}_{2} \mathrm{O}_{3}$ & $\mathrm{Al}_{2} \mathrm{O}_{3}$ & $\mathrm{SO}_{3}$ & $\mathrm{MgO}$ & $\mathrm{CaO}$ & $\mathrm{Na}_{2} \mathrm{O}$ & $\mathrm{K}_{2} \mathrm{O}$ & $\mathrm{TiO}_{2}$ \\
20.47 & 3.48 & 4.41 & 3.33 & 2.49 & 61.43 & 0.14 & 0.55 & 0.30 \\
\hline
\end{tabular}

Anionic asphalt emulsion (with a solid content of $60 \%$ ) was supplied by Jiangsu Bote New Materials Co., Ltd. Its main production process can be summarized as follows: the asphalt was first heated to about $140^{\circ} \mathrm{C}$, and it was then turned into asphalt droplets following a mechanical dispersion. The anionic asphalt emulsion was finally produced through the interactions between the asphalt droplets, anionic emulsifier and water in a colloid mill. Figure.1 illustrates schematically the processes involved in the manufacture of the anionic asphalt emulsion used in the current study. 


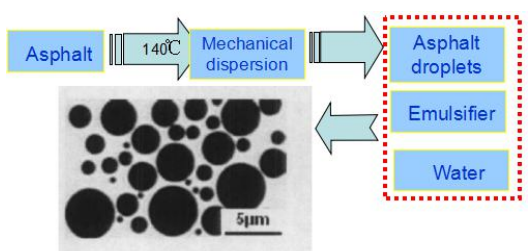

Figure.1 Schematic diagram of the production process of anionic asphalt emulsion

It should be noted that the anionic emulsifier is an essential component in the production of anionic asphalt emulsion. In this study, a lignin carboxylate emulsifier was employed [25], mainly due to the following two reasons: 1) the lignin carboxylate emulsifier could generate the negatively charged groups such as $-\mathrm{COO}^{-}$via ionization, which can enhance the dispersion stability of the anionic asphalt emulsion; 2) the negatively charged groups could be adsorbed onto the surface of the cement particles or the hydration products via the electrostatic attraction and this can improve the interaction between the cement particles and the asphalt emulsion. In addition, since the lignin carboxylate emulsifier is stable at high temperature, the anionic asphalt emulsion formulated with this type of emulsifier is, thus, suitable for producing cement asphalt pastes for a wider range of working temperatures.

\subsection{Sample preparation}

The CA pastes with different asphalt to cement $(\mathrm{A} / \mathrm{C})$ ratios, namely, $0,0.16,0.24$ and 0.32 (the mixes are hereafter denoted as CA- $0.16, \mathrm{CA}-0.24$ and CA-0.32, accordingly) were used to investigate the heat released during the early stage reaction of the CA pastes with an isothermal conduction calorimeter. Additionally, the electrical resistivity tests were carried out with $\mathrm{CA}$ pastes at a W/C of 0.41 and the $\mathrm{A} / \mathrm{C}$ of 0 and 0.24 in order to understand the effect of anionic asphalt emulsion on the evolution of the microstructure of CA pastes. It should be noted that the amount of the water in the anionic asphalt emulsion was also considered in the calculation of $\mathrm{W} / \mathrm{C}$, and the mix ratios were selected from the real engineering applications in China.

\subsection{Test methods}

\subsubsection{Setting time measurement}

Setting time was measured according to the standard of ISO 9597:2008--Cement-Test methodsDetermination of setting time and soundness, NEQ. Both the initial and final setting times of CA pastes with different anionic asphalt emulsion were measured, and the test temperature was kept at $20^{\circ} \mathrm{C}$.

\subsubsection{Hydration heat measurement}


The fresh CA pastes were put into a plastic bottle within 10 mins after mixing, and the heat released was then measured by a self-regulated isothermal conduction calorimeter. The temperature was equilibrated at $20^{\circ} \mathrm{C}$ between the specimen and the instrument before conducting the measurement, and the test was lasted for about 72 hours.

\subsubsection{Electrical resistivity measurement}

It has been well-established that the electrical resistivity can reflect the PC hydration process as well as the evolution of the microstructure during the hydration [26-28]. Therefore, a Non-Contact Impedance Measurement (NCIM) CCR- II (Figure.2) was employed to monitor the effect of asphalt emulsion on the hydration of PC and the evolution of the microstructure of CA pastes.

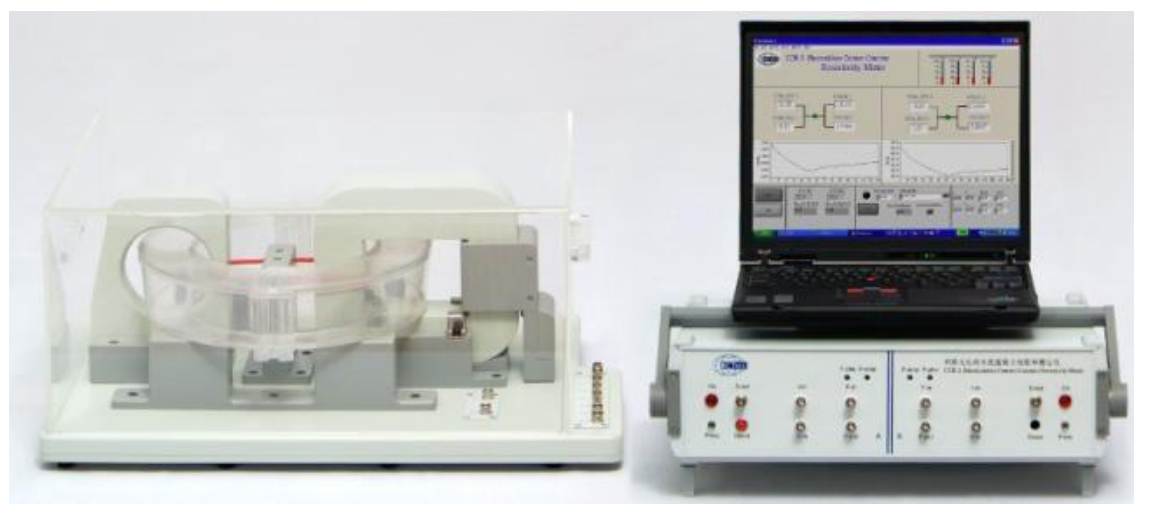

Figure.2 The Non-Contact Impedance Measurement employed in the investigation

Prior to any tests, the instrument was first adjusted to more than $30000 \Omega$. The fresh CA paste was then poured into the ring-shaped plastic mould and subsequently a series of automatic measurements were taken by the instrument. The basic steps involved in the operation can be summarized as follows: (1) A certain level of voltage was generated by the electro-magnetic induction via secondary generator after being charged with electricity. (2) The voltage then produced a ring current, which could be measured by the electric current transducer. (3) According to the Ohm's law, the electrical resistivity was calculated by measuring the height of the ring-shaped paste.

\subsubsection{MIP measurement}

The pore size distribution and the porosity of the PC and the CA pastes were measured by a Poremaster GT-60 mercury intrusion porosimeter (MIP) which can generate pressures from 140kPa to 420MPa. The porosimeter could be employed to measure the pore sizes from $0.0035 \mu \mathrm{m}$ to $400 \mu \mathrm{m}$.

Prior to the MIP test, the crushed pieces of the hardened samples obtained after the electrical resistivity test were firstly soaked in an absolute ethyl alcohol solution to stop the hydration. These 
specimens were then dried in a vacuum drying oven until a constant weight was reached, indicating the specimens were ready for the MIP measurement. The parameters employed for the MIP experiments are as follows: 1) the weight of samples was about $2 \mathrm{~g}$ with the particle sizes between $3-5 \mathrm{~mm}$;2) the maximun intrusion pressure was about 30000psia; 3) the value of contact angle was about $142^{\circ}$, and 4) the surface tension of mercury was $0.485 \mathrm{~N} / \mathrm{m}$ at $20^{\circ} \mathrm{C}$.

\subsubsection{SEM/ESEM observation}

Microstructural characterization of the hardened PC and CA pastes was carried out using a Scanning Electron Microscope (SEM), QUANTA 250 manufactured by FEI company. This device was equipped with a cold field emission electron gun operating at $15 \mathrm{kv}$. The samples for the SEM observation were the same as those for the MIP measurements, and the surface of the samples was sputter coated with a thin layer of Pt/Pd before the SEM observation.

To observe the adsorption behavior of anionic asphalt emulsion on the surface of cement grains during the hydration process, Environmental Scanning Electron Microscope (ESEM) was employed in the investigation. The test process can be summarized as follows: put the specimen on the cold platform in the interior of sample room after the preparation of the fresh CA paste well; cool down by starting the cold platform and keep a certain degree of vacuum, which in turn, ensure the equilibrium state between the three phases of moisture, air and liquid water, and thus maintain the hydration of cement during the measurement; and then the adsorption behavior could be observed by ESEM.

\section{Results}

\subsection{Influence of the anionic asphalt emulsion on the setting time}

Setting is a process whereby the cement particles start to connect with each other through the formation of hydration products. Hence, to certain extent, setting process can reflect the level of connectivity between the cement particles [29]. The setting time of cement paste is usually divided into initial setting time and final setting time. The initial setting time depicts the stage when the cement paste converts from a flowable state to the beginning of a hardening state, which is closely related to the rheological property of fresh paste; while the final setting time indicates the formation of hardened 
cement paste. Therefore, setting time has been considered as an important parameter to reflect the hydration process of PC during the early stages of hydration. However, in the presence of anionic asphalt emulsion, the hydration process of PC could become complex due to the interaction between the cement particles and the asphalt emulsion.

Figure. 3 shows that both the initial and the final setting times were prolonged with the increase of the $\mathrm{A} / \mathrm{C}$ from 0 to 0.32 , which implies that the $\mathrm{PC}$ hydration was retarded by the anionic asphalt emulsion. However, with the increase of the anionic asphalt emulsion, the gap between the initial setting and the final setting reduced.

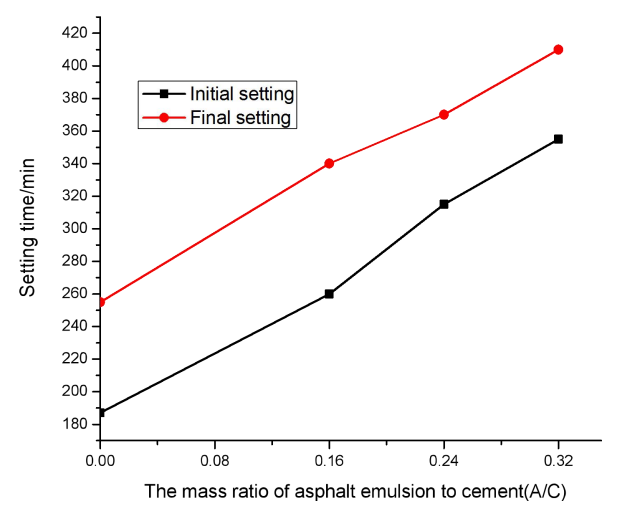

Figure. 3 Influence of the anionic asphalt emulsion on the setting times

The reduced gap between the initial and the final setting times could be attributed to the following reasons. As mentioned before, the initial setting refers to the conversion from a flowable state to a thixotropic state, while the final setting refers to the conversion from a thixotropic state to a hardened state. Therefore, the gap between the initial setting and the final setting is closely related to the duration of the thixotropy of the cement paste.It is well-established that the thixotropic state of the cement paste is strongly determined by the formation of the network structure, which in turn is mainly influenced by cement hydration, water to cement ratio and the volume fraction of the solid components in the matrix [29-30]. In the presence of the anionic asphalt emulsion, the surface of the cement particles could be covered by the asphalt membrane. Additionally, the agglomerates of asphalt particles could also be formed between the asphalt particles due to the demulsification of asphalt emulsion. In both cases, the formation of the network structure will be accelerated and, consequently, the duration of the thixotropy could also be reduced. This would explain the reduced the gap between the initial setting and the final setting as observed in Figure 3. 
In order to further understand the retardation caused by the anionic asphalt emulsion on the PC hydration process, the heat of hydration, electrical resistivity and pore structure were investigated and reported below.

\subsection{Influence of the anionic asphalt emulsion on the hydration heat}

It is well known that the hydration of PC involves the dissolution of different ions from the surface of cement particles upon in contact with water, resulting in a gradual increase in the concentration of ions over the hydration period. Once the ion concentration reaches the oversaturation level, the precipitation of the hydration products, such as $\mathrm{CH}$, Aft and C-S-H gel, starts. This process leads to the formation of much more stable chemical bonds compared to those in the anhydrous cement minerals, and thus heat would be released during the hydration process. In other words, cement hydration is an exothermal process which is always accompanied by the release of heat [29,31]. Hence, by following the evolution of the heat generated during the PC hydration, it is possible to identify the effect of asphalt emulsion on the PC hydration process. Figure.4(a) presents the heat flow during the first 70 hours of $\mathrm{PC}$ hydration at different $\mathrm{A} / \mathrm{C}$, whilst Figure.4(b) is an inset showing the details of heat evolution during the first 10 hours.

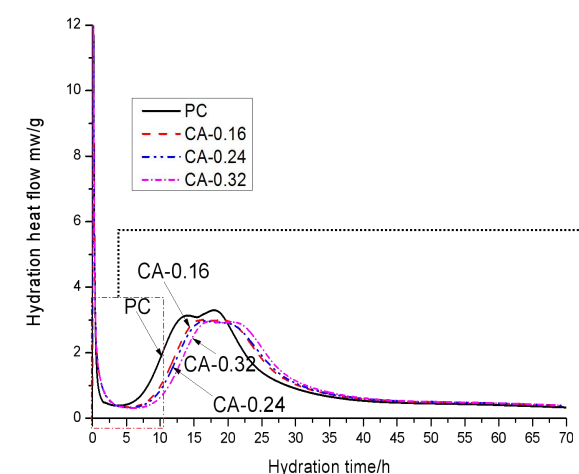

(a)

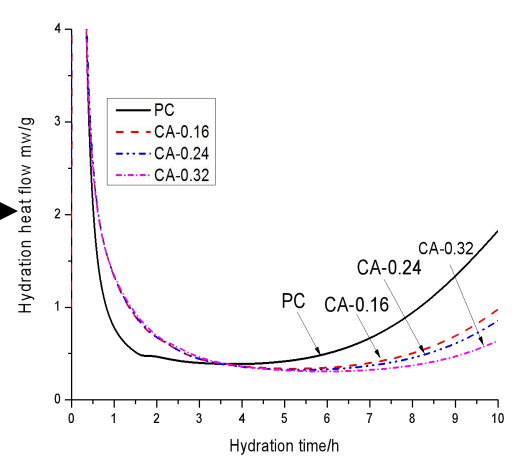

(b)

Figure.4 Influence of the anionic asphalt emulsion on the heat flow of PC hydration In terms of the $\mathrm{PC}$ hydration process, the ion dissolution rate of $\mathrm{C}_{3} \mathrm{~A}$ is much faster than that of the silicate phases, $\mathrm{C}_{2} \mathrm{~S}$ and $\mathrm{C}_{3} \mathrm{~S}$, due to the higher reactivity of aluminate phase, leading to a quick saturation of $\mathrm{Ca}^{2+}$ ions in the solution $[29,31-33]$. Hence, the initial hydration products such as Aft and C-S-H(m) are usually formed around the surface of cement grains, which would hinder the further dissolution from the anhydrous minerals and results in the initiation of the induction period. That is to 
say, the concentration of $\mathrm{Ca}^{2+}$ increases slowly over time during the induction period, and the induction period should be ended when the cumulative concentration of $\mathrm{Ca}^{2+}$ reaching certain level of saturation followed by the formation of large amount of C-S-H gel [29]. Therefore, the duration of the induction period mainly depends on the concentration of $\mathrm{Ca}^{2+}$, i.e., the quicker the increase of the concentration of $\mathrm{Ca}^{2+}$, the shorter the duration of the induction period of PC hydration [28,34].

From Figure.4(b), it can be seen that the induction period was prolonged with the increase of the $\mathrm{A} / \mathrm{C}$. It is generally agreed that the surface of the aluminate phase and certain part of the silicate phases show positive charge after mixing with water, which is occurred mainly due to the different migration rate of individual ions moving from the cement particles into the solution $[16,25]$. Hence, the anionic asphalt emulsion can be adsorbed onto the positively charged sites of cement particles through electrostatic interaction. Consequently, the surface of cement particles could be covered by an asphalt membrane, which would hinder the further dissolution of cement particles and resulted in the slow increase of the cumulative concentration of $\mathrm{Ca}^{2+}$ with the increase of $\mathrm{A} / \mathrm{C}$ during the induction period. Therefore, the duration of the induction period was prolonged due to the lower concentration of $\mathrm{Ca}^{2+}$ in the presence of anionic asphalt emulsion, i.e., the PC hydration process was retarded by the anionic asphalt emulsion.

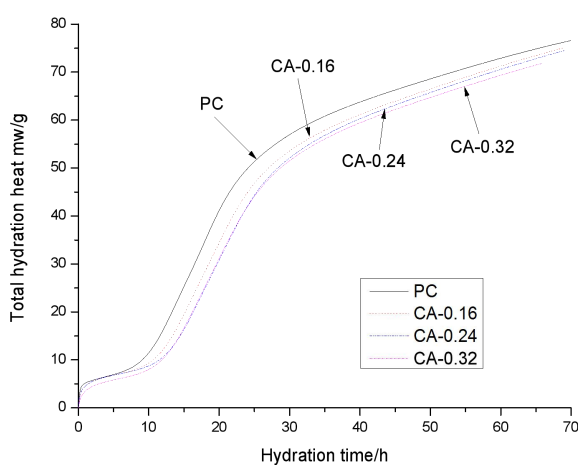

Figure.5 Influence of the anionic asphalt emulsion on the total cumulative hydration heat

As discussed in the previous section, the heat of hydration is generated from the formation of hydration products, and the total cumulative hydration heat can reflect the amount of hydration products formed, i.e. the total cumulative hydration heat could be used to indicate the degree of the hydration. In comparison to the pure PC paste, it can be seen from Figure 5 that the total cumulative hydration heat of the CA pastes containing different amounts of anionic asphalt emulsion reduced, which meant that the degree of the hydration of PC was decreased by anionic asphalt emulsion. 
1 Although the cement percentage in the CA samples was indeed lower than that of the pure PC sample,

2 the comparison of the total hydration heat is the amount of hydration heat released by per weight of cement without considering the other components. In other words, the total hydration heat refers to the cumulative hydration heat released from per weight of cement. Therefore, the reduced total hydration heat of the CA samples indicates that the cumulative hydration heat released per weight of cement was reduced in the presence of asphalt emulsion. This result further confirmed the retardation effect of the anionic asphalt emulsion on PC hydration.

\subsection{Influence of the anionic asphalt emulsion on the electrical resistivity}

The electrical resistivity has been widely used to follow the evolution of the hydration process of cementitious materials over time $[26,35]$. Especially, during the very early stage of the hydration, the electrical resistivity has been found to be a useful tool to follow the dissolution process as the change of the electrical resistivity is mainly dominated by the variation of the ion concentration in the solution, whilst at later stage, the electrical resistivity can reflect the evolution of the microstructure because, at that stage, it is the porosity and the connectivity of the pores which will dominate the electrical resistivity. In this study, the electrical resistivity was, thus, employed to investigate the evolution of the early-stage hydration process of the CA paste. Figure.6 shows the evolution of the electrical resistivity of the CA paste at an $\mathrm{A} / \mathrm{C}$ of 0.24 in comparison with the pure PC paste within the first 24 hours.

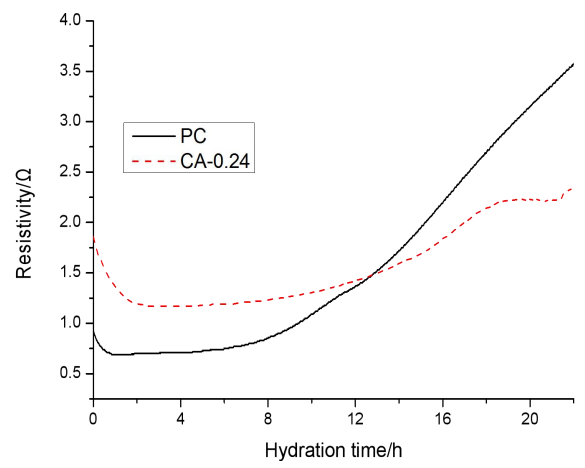

Figure.6 Influence of the anionic asphalt emulsion on the electrical resistivity of

$$
\mathrm{PC} \text { and } \mathrm{CA}-0.24 \text { pastes }
$$

It can be seen that the electrical resistivity of the pure PC paste and the CA paste exhibited a similar pattern, i.e., immediately after in contact with water, there is an immediate decrease of the electrical resistivity, which is then followed by a relatively constant resistivity for certain time before it starts to increase. These three clearly distinguishable stages of the variation in electrical resistivity can 
1 be well linked with the three well-established periods during their early hydration process, namely, the 2 dissolution period, the induction period and the acceleration period. It is evident that, during the dissolution period, the electrical resistivity of both the CA paste and the pure PC paste reduced quickly to a minimum value which lasted for about $100 \mathrm{~min}$ and $50 \mathrm{~min}$ respectively. Additionally, it can be noticed that the electrical resistivity of the CA paste was higher than that of the pure PC paste during the dissolution and induction periods whilst the opposite can be observed during the acceleration period. It is well known that, during the early dissolution stage, the electrical resistivity of fresh PC paste is mainly determined by the ion concentration in the fresh paste which, in turn, is controlled by the dissolution process. That is, the electrical resistivity of the fresh PC paste decreases with the increase of the ion concentration in solution. Therefore, the higher electrical resistivity of the CA paste during the dissolution and induction periods in Figure.6 would, once again, support the hypothesis proposed before that the surface of the cement grains could have been coated by asphalt emulsion, and this can lead to a reduced dissolution of the cement particles, resulting in a lower ion concentration and, hence, a higher electrical resistivity. Therefore, in comparison to the PC paste, the electrical resistivity of the CA paste was higher and it took much longer to reach the induction stage, i.e. the decline stage of the electrical resistivity lasted much longer, during the early dissolution stage.

After the early dissolution period, the electrical resistivity showed a pattern which is similar to the induction period of cement hydration. During this period, the electrical resistivity was nearly constant until the end of the induction period. Moreover, the induction period of CA paste lasted longer than that of the pure PC paste as shown in Figure.6, which could also be attributed to the reduced dissolution rate of CA paste due to the asphalt coating formed on the surface of the cement grains.

On the contrary, during the acceleration hydration period, the electrical resistivity of the CA paste was lower than that of the pure PC paste, which would indicate that the microstructure formed in the CA paste was more porous than that of the PC paste. This, again, could be attributed to the barrier formed by the asphalt membrane on the surface of cement grains which could have adversely affected the dissolution process of cement and, hence, retarded the hydration process and the formation of the microstructure of the CA paste.

3.4 Microstructure characterization 


\subsubsection{Pore structure analysis}

In order to identify the effect of anionic asphalt emulsion on the pore structure of the hardened CA paste, MIP was employed to characterize the pore structure of the pure PC paste and the CA paste at the age of $1 \mathrm{~d}$ as shown in Figure.7, and Figures.7 (a) and (b) present the pore size distribution and the cumulative pore volume, respectively. From Figure.7(a), it can be clearly seen that the pore size distribution of the PC paste is rather distributed, while the pore size distribution of the CA paste is more concentrated; in addition, the pore size of the PC paste is mainly dominated by smaller sizes, whereas the pore size of the CA paste shifted to the bigger sizes in comparison to that of PC paste. Figure.7(b) shows that the cumulative volume of the CA paste is higher than that of the PC paste, which indicates that the anionic asphalt emulsion resulted in the increase of the pore volume. Overall, it can be concluded that the CA paste is more porous, with much larger pores, than that of the PC paste.

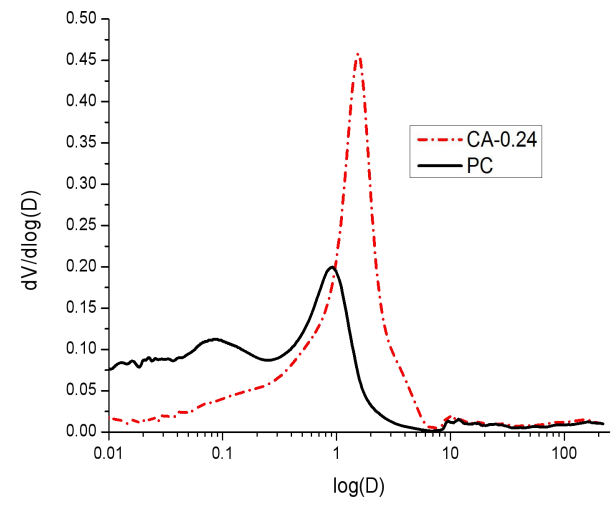

(a)

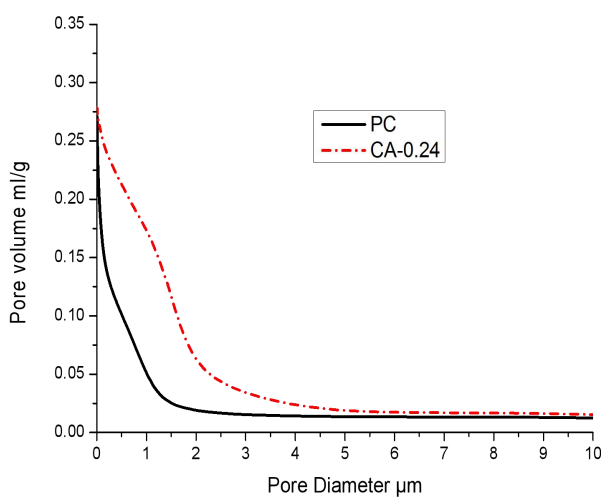

(b)

Figure.7 Pore size distribution and cumulative volume of the PC and CA- 0.24 pastes at the age of $1 \mathrm{~d}$ 3.4.2 Morphology analysis

With the continued hydration of the PC, the porosity of the PC paste decreases gradually, accompanied by the increased growth of the cement hydration products, leading to an increase in the electrical resistivity of the PC paste. Hence, there should exist an intrinsic relationship between the electrical resistivity and the microstructure of the PC and the CA paste. In this study, the microstructure of the hardened pure PC paste and the CA paste was observed by SEM and this is shown in Figure.8. From Figure.8, it is evident that the pure PC paste has a denser structure than that of the CA paste. In addition, much more hydration products can be observed to be connected with each other in the PC paste, resulting in the pores being filled in by the hydration products which, in turn, caused the 
1 formation of much more denser structure. In contrast, the CA paste showed relatively less hydration

2 products in comparison to the PC paste, and the CA paste exhibited more porous microstructure 3 because the pores in the CA paste was not filled in by large amounts of the hydration products, leading to the formation of more large pores in the CA paste.

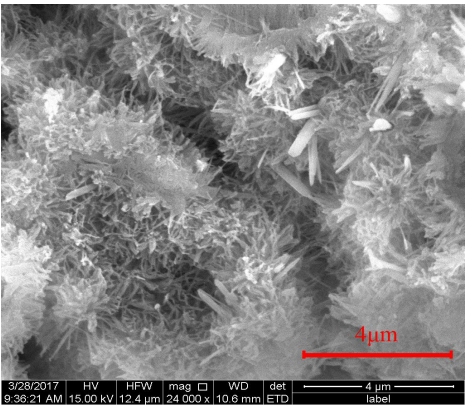

(a)

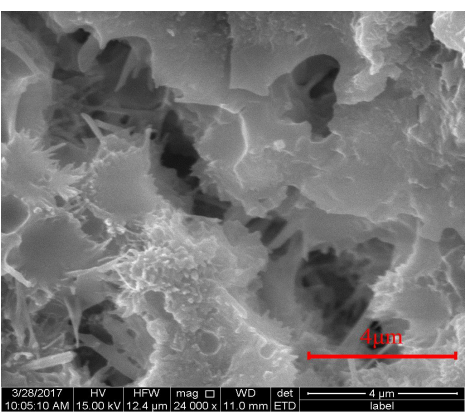

(b)

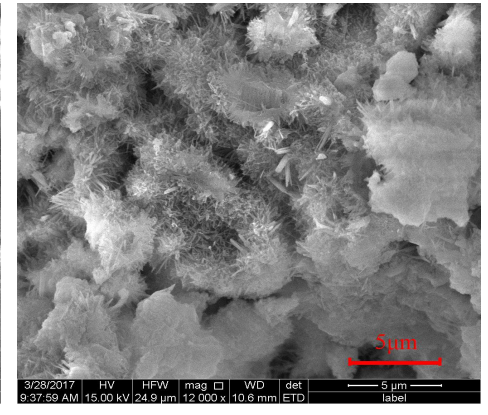

(c)

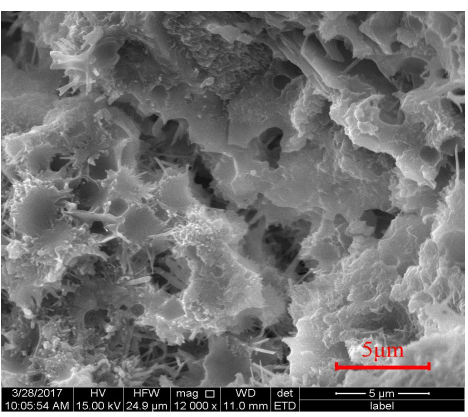

(d)

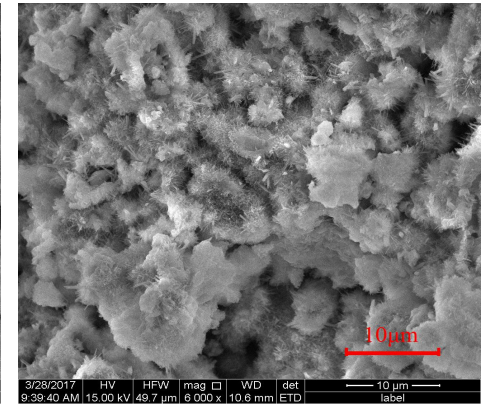

(e)

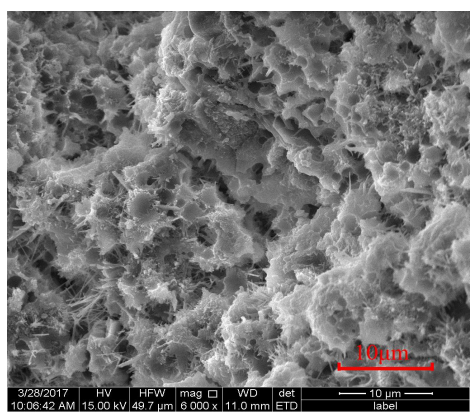

(f)

Figure. 8 Microstructure of the hardened pure PC paste and CA-0.24 paste at the age of 1d: (a) PC paste magnified at 24000 times; (b) CA-0.24 paste magnified at 24000 times; (c) PC paste magnified at 12000 times; (d) CA-0.24 paste magnified at 12000 times; (e) PC paste magnified at 6000 times; (f) CA-0.24 paste magnified at 6000 times.

Combining the results from the MIP and the SEM, it can be concluded that the CA paste was more porous than that of the pure PC paste and this would explain the lower electrical resistivity measured from the hardened CA paste which could, in turn, reflect the fact that the PC hydration was retarded by the anionic asphalt emulsion.

\section{Discussion}

Based on the results presented in Section 3, three possible retardation mechanisms introduced by the anionic asphalt emulsion on the hydration and the microstructure evolution of the PC is assessed and discussed below. 
4.1 Mechanism |---Selective adsorption by anionic emulsifier via electrostatic attraction

It is well established that immediately upon being in contact with water, different mineral phases or hydration products of PC would demonstrate different charges. Specifically, the aluminate phase, $\mathrm{C}_{3} \mathrm{~A}$, usually shows a positive charge with a zeta potential of $+4.15 \mathrm{mv}$, while the silicate phases, $\mathrm{C}_{2} \mathrm{~S}$ and $\mathrm{C}_{3} \mathrm{~S}$, show negative charge [36]. However, certain part of the silicate phases may also show positive charge due to the complexation existing between the calcium ions, $\mathrm{Ca}^{2+}$, and the $\mathrm{H}_{2} \mathrm{SiO}_{4}{ }^{2-}$ around the surface of silicate phases via electrostatic attraction [16].

The anionic asphalt emulsion used in this study was negatively charged because the anionic emulsifier was used to emulsify the asphalt. According to reference [16], negative charge is the driving force for the adsorption onto the positively charged sites on the surface of cement particles. Therefore, anionic asphalt emulsion can be selectively adsorbed onto those positively charged sites on the surface of cement grains, such as those sites occupied by aluminate phase or certain part of silicate phases, through anionic emulsifier via electrostatic adsorption [37-38]. This layer of adsorbed anionic asphalt emulsion would then hinder the further dissolution of anhydrous phases as well as the subsequent deposition of hydration products on the surface of cement particles, which may be one of the retardation mechanisms that the anionic asphalt emulsion applied to the hydration of PC. Figure.9 further illustrates this adsorption behavior of anionic asphalt emulsion onto the cement particles.

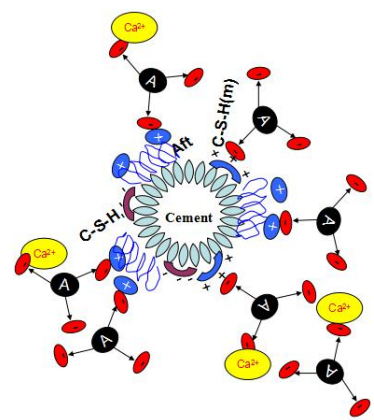

Figure.9 Schematic diagram showing the selective adsorption by anionic emulsifier via electrostatic attraction 4.2 Mechanism II--- Coating formed by the demulsification of anionic asphalt emulsion Asphalt emulsion is a thermodynamically unstable system, which maintains its dispersion through the electrostatic repulsion between the double electrode layers. However, once the counter ions, such as $\mathrm{Ca}^{2+}$, are dissolved from cement particles and diffused into the double electrode layers of anionic 
1 asphalt emulsion, or due to the consumption of water during cement hydration, the asphalt emulsion

2 could demulsify, resulting in the aggregation of asphalt particles and the formation of asphalt membrane [39]. As a result, further dissolution and deposition reactions could be hindered due to the asphalt membrane coating formed on the surfaces of cement particles (as shown in Figure.10). This could be another possible retardation mechanism that the anionic asphalt emulsion applied to the PC hydration. To verify this hypothesis, an Environmental Scanning Electron Microscope (ESEM) was employed to observe the adsorption behavior of the anionic asphalt emulsion onto the cement particles during its early hydration stage [40]. From Figure.11, it can be clearly seen that the surface of the cement particles was covered by asphalt membrane (possibly due to the demulsification of asphalt emulsion), which further confirmed the above hypothesis of the interaction between cement particles and anionic asphalt emulsion.

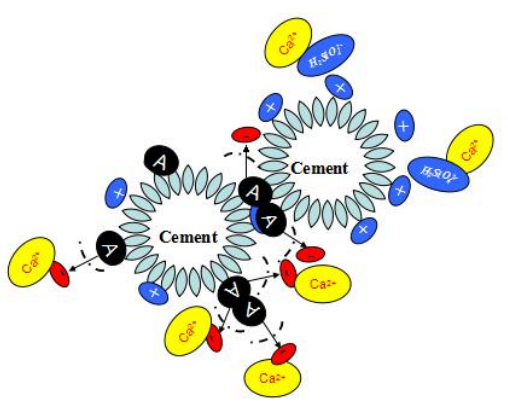

Figure.10 Schematic diagram of the asphalt membrane formed on the surfaces of cement particles
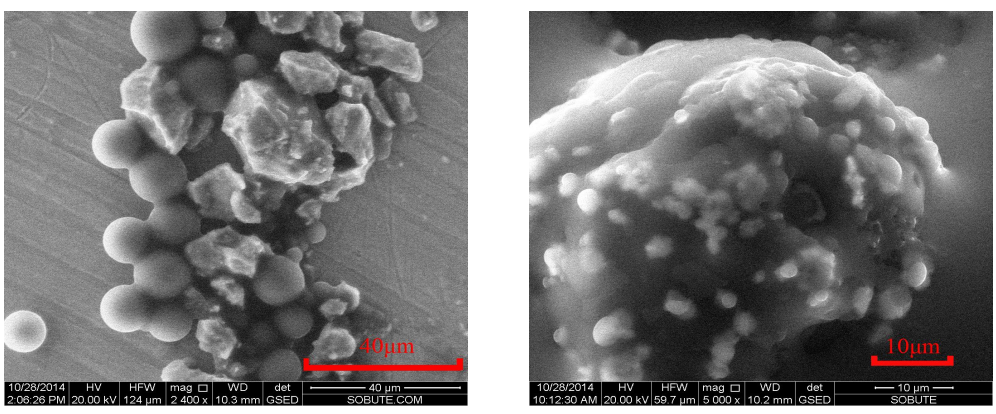

Figure.11 ESEM micrograph showing the adsorption of anionic asphalt emulsion onto cement particles

Therefore, it can be seen that both the above two mechanisms can lead to the hindrance of ion dissolution from the surface of the cement particles and thus retarded the cement hydration process. However, mechanism I is mainly about the selective adsorption by anionic emulsifier, while mechanism II is mainly about the coverage of the cement particles by asphalt membrane. There are some distinguishable differences between these two mechanisms. The driving force for the selective adsorption is the electrostatic attraction between the anionic emulsifier and the positively charged sites 
1 on the surface of cement particles, and this selective adsorption mainly results in some positively

2 charged sites of cement particles being occupied by anionic emulsifier but not asphalt particles at this

3 stage. Additionally, this adsorption mechanism mainly dominates during the early period of the reaction before the demulsification of asphalt emulsion occurs. However, with the continuous hydration of cement and, thus, further consumption of water, the asphalt emulsion adsorbed selectively onto the surface of cement particles would demulsify, resulting in the aggregation of the asphalt particles and the formation of the coverage of asphalt coating onto the whole cement particles. This can further hinder the ion dissolution and subsequent deposition reaction of cement.

4.3 MechanismIII---Reduced reactivity of water molecules in anionic asphalt emulsion

One additional retardation mechanism which could be introduced by the anionic asphalt emulsion to the hydration of PC may be related to the reactivity of water molecules in the anionic asphalt emulsion. As well known, water molecule is a kind of polar molecule. The hydrogen atom in water molecule is short of electrons, while the oxygen atom in water molecule possesses extra electrons. Therefore, hydrogen bonds can be formed between the hydrogen atom and the oxygen atom from different water molecules. Hence, the structure of liquid water usually can be seen as a continuous network of hydrogen bonds formed between different water molecules [41-42]. Figure.12 presents the schematic structure of the hydrogen bonds (as denoted by dashed line in red) formed between different water molecules. This structure makes the distribution of electronic clouds in a much more equilibrated status, and thus decreased the energy of the water molecules, i.e., the reactivity of water molecules decreases with the formation of more hydrogen bonds [42].

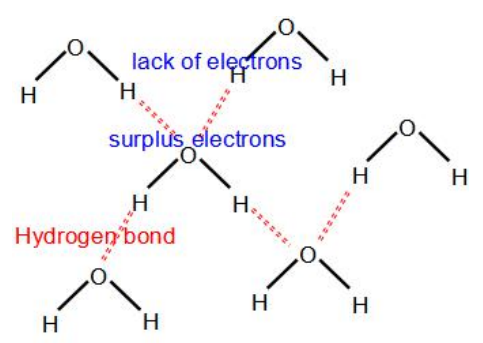

Figure.12 Schematic of the hydrogen bonds formed between water molecules

According to the perspective of crystal, the silicate phase of $\mathrm{C}_{3} \mathrm{~S}$ can be considered as an ionic crystal composed of $\mathrm{Ca}^{2+}$ cations with oxide and monomeric silicate anions $\left(3 \mathrm{Ca}^{2+} \cdot \mathrm{O}^{2-\cdot} \cdot \mathrm{SiO}_{4}{ }^{4-}\right)$, which can be ionized after contacting with water, and consequently can be converted from the superficial 
oxide ions to hydroxide ions due to the instability of hydrogen ions in water molecules. In other words, the initial PC hydration could be regarded as a process whereby the hydrogen atom in water molecule attacks and breaks down the $\mathrm{Al}-\mathrm{O}$ and $\mathrm{Ca}-\mathrm{O}$ bonds on the surface of cement particles, resulting in the formation of much more stable bond of $\mathrm{OH}^{-}$because the energy of $\mathrm{H}-\mathrm{O}$ bond is much higher than that of the Al-O and Ca-O [43-46]. As aforementioned, the reactivity of water with cement is closely related to the unequilibrated distribution of the electronic clouds in water molecules. More hydrogen bonds can lead to a more equilibrated distribution of the electronic clouds between the water molecules, which would decrease the reactivity of water molecules with cement particles. Figure. 13 presents the reaction mechanism of water molecule with cement particle.

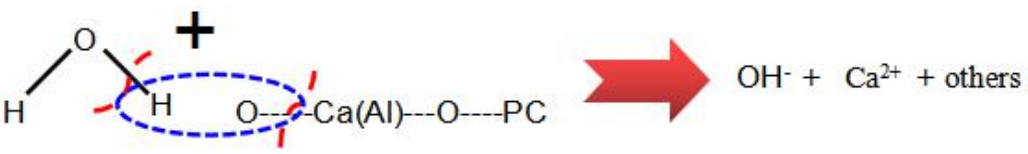

Figure.13 Reaction mechanism between water molecule and cement particles Anionic asphalt emulsion is composed of anionic emulsifier and water. The anionic emulsifier is a hydrophilic group which possesses extra electrons, while the hydrogen atom in water molecule is short of electrons. Therefore, hydrogen bonds would be preferentially formed between the hydrogen atom in water molecule and the hydrophilic group of the anionic emulsifier, which could cause the electronic clouds distribution between the oxygen atom and the hydrogen atom in water molecule became unequilibrated, and thus results in the formation of more hydrogen bonds between different water molecules in anionic asphalt emulsion[47-50]. In addition, anionic asphalt emulsion is a kind of colloid system with the size of less than $5 \mu \mathrm{m}$, which could be seen as a solution composed of lots of colloid centers. In other words, hydrogen bonds would be formed between the water molecules around every colloid center, which means that much more hydrogen bonds would be formed between water molecules in anionic asphalt emulsion than in the pure water molecules [51-53]. A schematic illustration of the hydrogen bonds formed between the water molecules around the anionic asphalt emulsion is presented in Figure.14. Therefore, the reactivity between water and cement particles could have been reduced due to the formation of much more hydrogen bonds between the water molecules in anionic asphalt emulsion[42], which may be considered as another retardation mechanism of anionic asphalt emulsion on PC hydration. 


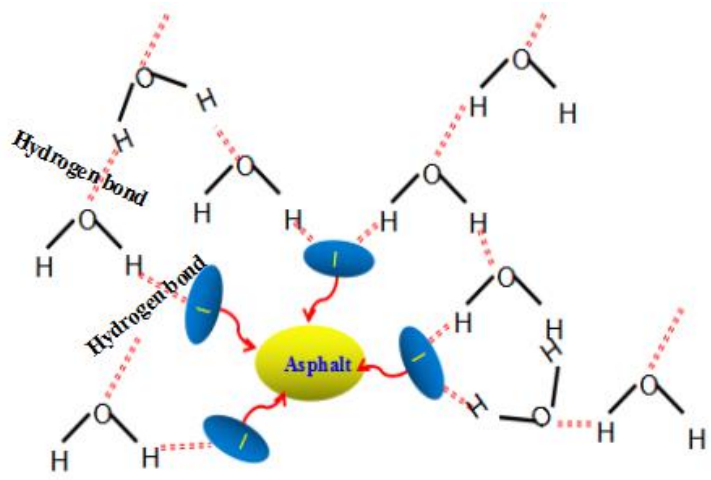

Figure.14 Schematic illustration of the hydrogen bonds formed around anionic asphalt emulsion

\section{Conclusions}

This study clearly demonstrated that PC hydration was retarded by the anionic asphalt emulsion. Based on the experimental results, three possible retardation mechanisms introduced by the anionic asphalt emulsion on the PC hydration were assessed. The main conclusions of this investigation can, thus, be drawn as follows:

(1) The initial and the final setting times were prolonged gradually with the increase of A/C from 0 to 0.32 , which implied that PC hydration was retarded by the anionic asphalt emulsion.

(2) The release of hydration heat was delayed with the increase of $\mathrm{A} / \mathrm{C}$ during the induction period, and meanwhile the total cumulative hydration heat of CA paste was lower than that of the PC paste, which indicated that the degree of the PC hydration was decreased by the anionic asphalt emulsion.

(3) During the early dissolution period, the electrical resistivity of fresh CA paste was higher than that of the pure PC paste, which meant that the ion dissolution process was hindered by the anionic asphalt emulsion in the CA paste. However, during the accelerated hydration period, the electrical resistivity of hardened CA paste was lower than that of the pure PC paste due to the higher porosity.

The results of SEM and MIP verified that the CA paste was more porous than the pure PC paste, which implied that the formation of the microstructure of the CA paste was also retarded by the anionic asphalt emulsion.

(4) Three retardation mechanisms introduced by the anionic asphalt emulsion on PC hydration have been proposed and assessed, including: (i) selective adsorption by anionic emulsifier via 
1 electrostatic attraction, (ii) coating formed by the demulsification of anionic asphalt emulsion, and (iii)

2 reduced reactivity of water molecules in anionic asphalt emulsion.

3

\section{Acknowledgements}

The authors are greatly grateful for the supports received from the National Natural Science Foundation of China (NO.51708483), National Natural Science Foundation of China (NO.51578479) and National Science \& Technology Pillar Program(2014BAB15B01-02). The first author also wants to thank the Advanced \& Innovative Materials (AIM) Group at University College London (UCL) in the UK and the State Key Laboratory of High Performance Civil Engineering Materials in China for funding this project.

\section{References}

[1]Turatsinze A, Bonnet S, Granju J L. Mechanical characterization of cement-based mortar incorporating rubber aggregates from recycled worn tyres. Building \& Environment, 2005, 40(2):221-226.

[2] H. Shoukry, M.F. Kotkata, S.A. Abo-el-Enein et al.Flexural strength and physical properties of fiber reinforced nano metakaolin cementitious surface compound. Constr Build Mater. (2013) 43:453-460.

[3] Karihaloo B L, Carpinteri A, Elices M. Fracture mechanics of cement mortar and plain concrete. Advanced Cement Based Materials, 1993, 1(2):92-105.

[4] Craeye B, Itterbeeck P V, Desnerck P, et al. Modulus of elasticity and tensile strength of self-compacting concrete: Survey of experimental data and structural design codes. Cement \& Concrete Composites, 2014, 54:53-61.

[5] Alsalman A, Dang C N, Prinz G S, et al. Evaluation of modulus of elasticity of ultra-high performance concrete[J]. Construction \& Building Materials, 2017, 153:918-928.

[6] Li S, Huang Y, Liu Z H. Experimental evaluation of asphalt material for interlayer in rigid flexible composite pavement[J]. Construction \& Building Materials, 2016, 102:699-705.

[7] Goh S W, Akin M, You Z, et al. Effect of deicing solutions on the tensile strength of micro- or nano-modified asphalt mixture[J]. Construction \& Building Materials, 2011, 25(1):195-200. 
1 [8] Cheng-Tsung Lu, Ming-Feng Kuo, Der-Hsien Shen.Composition and reaction mechanism of cement-asphalt mastic. Constr Build Mater. (2009) 23:2580-2585.

[9] G Li, Y Zhao, S-S Pang, W Huang. Experimental study of cement-asphalt emulsion composite. Cem Concr Res. (1998) 28(5):635-641.

[10] Rutherford T, Wang Z, Shu X, et al. Laboratory investigation into mechanical properties of cement emulsified asphalt mortar[J]. Construction \& Building Materials, 2014, 65(13):76-83.

[11] Qiang W, Yan P, Ruhan A, et al. Strength Mechanism of Cement-Asphalt Mortar[J]. Journal of Materials in Civil Engineering, 2011, 23(9):1353-1359.

[12] Rong Chen, Ping Wang, Zhe Liu. Influences of CA Mortar Elastic Modulus on Stress and Deformation of Slab Track in High Speed Railway. Advanced materials research. (2011) vol (197-198): 1480-1485.

[13] Esveld C .Recent developments in slab track. Eur Railway Rev (2003) 2: 81-85.

[14] Wang Z, Wang Q, Yang G. Mechanism Analyses of Structure Formation of Cement Asphalt Emulsion Mixtures[J]. Advanced Materials Research, 2012, 374-377:799-802.

[15] Dan H E, Xiang J, Guo G J, et al. The effect of stiffness and damping of cement asphalt mortar on the vertical vibration of the high-speed train and slab track time-dependent system[J]. Journal of Railway Science \& Engineering, 2006.

[16] Johann Plank, Christian Hirsch.Impact of zeta potential of early hydration phases on superplasticizer adsorption. Cem Concr Res. (2007) 37: 537-542.

[17] Yiqiu Tan, Jian Ouyang, Jianfu Lv, et al. Effect of emulsifier on cement hydration in cement asphalt mortar. Constr Build Mater. (2013) 47:159-164.

[18] Ouyang J, Han B, Cao Y, et al. The role and interaction of superplasticizer and emulsifier in fresh cement asphalt emulsion paste through rheology study[J]. Construction \& Building Materials, 2016, 125:643-653.

[19]Chinese Railway Specification.Technical specification of CA mortar for CRTS non-ballast slab track. China:China Railway Publishing House; 2008,volume(a).

[20] Yanrong Zhang, Xiangming Kong, Shanshan Hou, et al. Study on the rheological properties of fresh cement asphalt paste. Constr Build Mater. (2012) 27:534-544. 
1 [21] Shaowen Du. Interaction mechanism of cement and asphalt emulsion in asphalt emulsion mixtures. Mater Struct. (2014) 47:1149-1159.

[22] Yang Jinbo, Yan Peiyu, Kong Xiangming, Li Xiang. Study on the hardening mechanism of cement asphalt binder. SCIENCE CHINA Technological Sciences. (2010) 53(5):1406-1412.

[23]Chinese Railway Specification.Technical specification of CA mortar for CRTS non-ballast slab track. China:China Railway Publishing House; 2008,volume(b).

[24]Wu Shaoliang, Li Honggang, Jia Hengqiong, et al. Comparative study on mechanical performance of two kinds of emulsified asphalt mortar used in high railway. Railway Engineering, 2015,3: 134136.

[25] Wei Li, Xiaobin Zhu, Jinxiang Hong, et al. Effect of anionic emulsifier on cement hydration and its interaction mechanism. Constr Build Mater.(2015) 93:1003-1011.

[26] Xiao Lianzhen.Interpretation of hydration process of concrete based on electrical resistivity measurement. The Hong Kong University of Science and Technology, Thesis of Doctoral (2007), Hong Kong.

[27] S.W. Tang, Z.J. Li, H.Y. Shao, et al. Characterization of early-age hydration process of cement pastes based on impedance measurement. Constr Build Mater. (2014) 68:491-500.

[28] X. Wei, Z. Li.Study on hydration of Portland cement with fly ash using electrical measurement. Mater Struct. (2005) 38:411-417.

[29] J.Bensted, P.Barnes. Structure and Performance of Cements, the 2nd Edition. (2008) Published by Taylor \& Francis.

[30] Flatt R J, Bowen P. Yodel: A Yield Stress Model for Suspensions[J]. Journal of the American Ceramic Society, 2006, 89(4):1244-1256.

[31] Fernández M M C. Effect of Particle Size on the Hydration Kinetics and Microstructural Development of Tricalcium Silicate, PhD dissertation. 2008.

[32] Scrivener K L, Juilland P, Monteiro P J M. Advances in understanding hydration of Portland cement. Cement and Concrete Research, 2015, 78:38-56.

[33]Bullard J W, Jennings H M, Livingston R A, et al. Mechanisms of cement hydration. Cement and Concrete Research, 2011, 41(12):1208-1223. 
1 [34]Kong X, Pakusch J, Jansen D, et al. Effect of polymer latexes with cleaned serum on the phase development of hydrating cement pastes. Cement and Concrete Research, 2016, 84:30-40.

[35]Zongjin Li. Advanced Concrete Technology[M]. Wiley, 2011.

[36] H. Viallis-Terrisse, A. Nonat, J. -C. Petit. Zeta-potential study of calcium silicate hydrates interacting with alkaline cations. J. Colloid Interface Sci. (2001) 244: 58-65.

[37] K.Yoshioka, E.-I. Tazawa, K.Kawai, et al. Adsorption characteristics of superplasticizers on cement component minerals. Cement and Concrete Research,(2002) 32:1507-1513.

[38] Kong X, Shi Z, Lu Z. Synthesis of novel polymer nano-particles and their interaction with cement. Construction \& Building Materials, 2014, 68(4):434-443.

[39] Jian Ouyang, Yiqiu Tan, Yunliang Li, et al. Demulsification process of asphalt emulsion in fresh cement-asphalt emulsion paste. Mater Struct. (2015) 48:3875-3883.

[40] M. Gretz, J. Plank. An ESEM investigation of latex film formation in cement pore solution. Cement and Concrete Research,(2011) 41:184-190.

[41] Jing Guo, Jingtao Lu, Yexin Feng, et al. Nuclear quantum effects of hydrogen bonds probed by tip-enhanced inelastic electron tunneling. Science. (2016) 352(6283): 321-325.

[42] Ran Cai, Hongwei Yang, Jinsong He, Wanpeng Zhu. The effects of magnetic fields on water molecule hydrogen bonds. Journal of Molecular Structure. (2009) 938: 15-19.

[43]Pustovgar E, Sangodkar R P, Andreev A S, et al. Understanding silicate hydration from quantitati-ve analyses of hydrating tricalcium silicates. Nature Communications, 2016, 7:10952.

[44]Mishra R K, Flatt R J, Heinz H. Force Field for Tricalcium Silicate and Insight into Nanoscale Properties: Cleavage, Initial Hydration, and Adsorption of Organic Molecules. Journal of Physical Chemistry C, 2013, 117(117):10417-10432.

[45]Li Beixing, Yu Qijun, Feng Xiuji. SCC-DV-X $\alpha$ Studies on difference in hydration activity of calcium aluminate minerals in CaO-A12O3 system. Journal of the Chinese Ceramic Society, 1998, 26(4):411-416.

[46] Zhou Y, Hou D, Manzano H, et al. Interfacial Connection Mechanisms in Calcium-SilicateHydrates/ Polymer Nanocomposites: A Molecular Dynamics Study. Acs Appl Mater Interfaces, 2017,9:41014-41025. 
1 [47]Xia G, He L, Zhao Q, et al. In-situ observation of the crystallization of Nylon-6 mediated by the interfacial hydrogen bonds. Colloid Polym Sci. Colloid \& Polymer Science, 2012, 290(18): 1943 1947.

[48]Khusnutdinoff R M. Dynamics of a network of hydrogen bonds upon water electrocrystallization. Colloid Journal, 2013, 75(6):726-732.

[49]Bus J, Groeneweg F, Vader F V V. Effect of hydrogen bonding on water in oil emulsion properties. Surfactants and Macromolecules: Self-Assembly at Interfaces and in Bulk. Steinkopff, 1990:122130.

9 [50] Tausk R J M, Wilson P N. Colloid chemical studies on bitumen-in-water emulsions part I. Absorption of water in the bitumen droplets and other factors affecting emulsion viscosity. Colloids \& Surfaces, 1981, 2(1):71-80.

[51] Furlong S, James A, Kalinowski E, et al. Water enclosed within the droplets of bitumen emulsions and its relation to viscosity changes during storage. Colloids \& Surfaces A Physicochemical \& Engineering Aspects, 1999, 152(1-2):147-153.

[52] Wan Zhongyi. Synthesis and performance characterization of novel cationic asphalt emulsifier. Shandong University, Thesis of Master (2008), Jinan, China.

[53] Al-Sabagh A M. The relevance HLB of surfactants on the stability of asphalt emulsion. Colloids \& Surfaces A Physicochemical \& Engineering Aspects, 2002, 204(1-3):73-83. 